PROCEEDINGS OF THE AMERICAN MATHEMATICAL SOCIETY

Volume 124, Number 8, August 1996

\title{
NICELY GENERATED AND CHAOTIC IDEALS
}

\author{
JÖRG BRENDLE \\ (Communicated by Andreas R. Blass)
}

\begin{abstract}
We show that if the real line is the disjoint union of $\kappa$ meager sets such that every meager set is contained in a countable union of them, then $\kappa=\omega_{1}$. This answers a question addressed by Jacek Cichoń. We also prove two theorems saying roughly that any attempt to produce the isomorphism type of the meager ideal in the Cohen real and the random real extensions must fail. All our results hold for meager replaced by null, as well.
\end{abstract}

\section{INTRODUCTION}

The purpose of this note is to prove three results concerning the structure of the $\sigma$-ideals of meager sets $\mathcal{M}$ and of null sets $\mathcal{N}$ on the reals. By the reals we shall mean the Cantor space $2^{\omega}$, equipped with the product topology and the product measure (where 2 carries the discrete topology and the measure giving both 0 and 1 measure $\left.\frac{1}{2}\right)$.

In the first section we investigate ideals which are generated by small unions of sets in a partition of the underlying set. We show that - except for trivial cases the meager and null ideals are not of this form (Corollary 6 ). In the second section we study the structure of the meager ideal $\mathcal{M}$ and the null ideal $\mathcal{N}$ in both the Cohen and random real models. Unfortunately, all our results (Theorems $9,9^{\star}, 10$, $\left.10^{\star}\right)$ are on the non-structural side.

Notational remarks. Given cardinals $\kappa$ and $\lambda,[\lambda]^{<\kappa}$ denotes the set of subsets of $\lambda$ of size $<\kappa$ and $[\lambda]^{\kappa}$ stands for the set of subsets of $\lambda$ of size $\kappa$. Let $\mathcal{B}$ denote the collection of Borel subsets of $2^{\omega}$. Then $\mathbb{C}=\mathcal{B} / \mathcal{M}$ is the Cohen algebra, and $\mathbb{B}=$ $\mathcal{B} / \mathcal{N}$ is the random algebra. More generally, for a cardinal $\kappa, \mathbb{C}_{\kappa}\left(\mathbb{B}_{\kappa}\right.$, respectively) is the algebra adding $\kappa$ Cohen (random, resp.) reals; and for $A \subseteq \kappa, \mathbb{C}_{A}\left(\mathbb{B}_{A}\right.$, resp.) is the complete subalgebra adjoining the $|A|$ many Cohen (random, resp.) reals with index in $A$. When talking about an ideal $\mathcal{I}$ on a set $X$ (i.e. $\mathcal{I} \subseteq P(X)$ ), we assume throughout that $\bigcup \mathcal{I}=X$ and $X \notin \mathcal{I}$ to avoid pathologies. For more set-theoretical notation we refer the reader to [Je] or [Ku 1]. For the basic facts about Cohen and random forcing see [Ku 2].

Received by the editors July 15, 1994 and, in revised form, February 23, 1995.

1991 Mathematics Subject Classification. Primary 03E05, 03E40, 28A05, 54H05.

Key words and phrases. Meager sets, null sets, Cohen reals, random reals.

Supported by DFG-grant Nr. Br 1420/1-1.

(C)1996 American Mathematical Society 


\section{NICELY GENERATED IDEALS}

Definition 1. Let $X$ be a set, and let $\kappa \leq \lambda$ be cardinals with $\lambda \leq|X|$. An ideal $\mathcal{I} \subseteq P(X)$ is $(\kappa, \lambda)$-nicely generated if there is a partition $\left\langle X_{\alpha} \in \mathcal{I} ; \alpha<\lambda\right\rangle$ of $X$ such that

(i) $\bigcup_{\alpha \in \Delta} X_{\alpha} \in \mathcal{I}$ for any $\Delta \in[\lambda]^{<\kappa}$ and

(ii) for any $Y \in \mathcal{I}$ there is $\Delta \in[\lambda]^{<\kappa}$ with $Y \subseteq \bigcup_{\alpha \in \Delta} X_{\alpha}$.

In this section we try to characterize the situations in which $\mathcal{M}$ and $\mathcal{N}$ can be nicely generated.

Definition 2. For an ideal $\mathcal{I} \subseteq P(X)$ we define the following cardinal characteristics:

$$
\begin{aligned}
& \operatorname{add}(\mathcal{I})=\min \{|\mathcal{F}| ; \mathcal{F} \subseteq \mathcal{I} \wedge \cup \mathcal{F} \notin \mathcal{I}\} \\
& \operatorname{cov}(\mathcal{I})=\min \{|\mathcal{F}| ; \mathcal{F} \subseteq \mathcal{I} \wedge \bigcup \mathcal{F}=X\} ; \\
& \operatorname{unif}(\mathcal{I})=\min \{|Y| ; Y \subseteq X \wedge Y \notin \mathcal{I}\} ; \\
& \operatorname{cof}(\mathcal{I})=\min \{|\mathcal{F}| ; \mathcal{F} \subseteq \mathcal{I} \wedge \forall Y \in \mathcal{I} \exists Z \in \mathcal{F}(Y \subseteq Z)\}
\end{aligned}
$$

It is well known which inequalities are provable in $Z F C$ between these cardinal invariants for $\mathcal{I}=\mathcal{M}$ and $\mathcal{I}=\mathcal{N}$ (see $[\mathrm{Fr}],[\mathrm{BJ}])$. Furthermore, their values in various $Z F C$-models have been studied intensively (see [BJS], [BJ]).

Fact 3. If $X, \kappa, \lambda$ are as in Definition 1 and $\mathcal{I} \subseteq P(X)$ is $(\kappa, \lambda)$-nicely generated, then

$$
\begin{aligned}
\operatorname{add}(\mathcal{I}) & =c f(\kappa), \\
\operatorname{cov}(\mathcal{I}) & = \begin{cases}\lambda & \text { if } \kappa<\lambda, \\
c f(\lambda) & \text { if } \kappa=\lambda,\end{cases} \\
\operatorname{unif}(\mathcal{I}) & =\kappa, \\
\operatorname{cof}(\mathcal{I}) & =\operatorname{cov}^{\star}(<\kappa, \lambda),
\end{aligned}
$$

where $\operatorname{cov}^{\star}(<\kappa, \lambda)=\min \left\{|\mathcal{F}| ; \mathcal{F} \subseteq[\lambda]^{<\kappa} \wedge \forall Y \in[\lambda]^{<\kappa} \exists Z \in \mathcal{F}(Y \subseteq Z)\right\}$.

The following result is well known. We include a proof for the sake of completeness.

Lemma 4 (Folklore). Assume $\left\{A_{\alpha} ; \alpha<\lambda^{+}\right\}$is a family of sets of size $<\lambda$. Then there are $\Lambda \in\left[\lambda^{+}\right]^{\lambda^{+}}$and a set $A$ of size $\leq \lambda$ such that $A_{\alpha} \cap A_{\beta} \subseteq A$ for distinct $\alpha, \beta \in \Lambda$. In case $\lambda$ is regular, we can find such $\Lambda$ and $A$ with $|A|<\lambda$.

Proof. Without loss $A_{\alpha} \subseteq \lambda^{+}$for $\alpha \in \lambda^{+}$. Let $\beta_{\alpha}<\lambda$ be the order type of $A_{\alpha}$ under the inherited ordering. Find $\Gamma \in\left[\lambda^{+}\right]^{\lambda^{+}}$and $\beta<\lambda$ with $\beta_{\alpha}=\beta$ for $\alpha \in \Gamma$. Let $\gamma<\beta$ be minimal such that $\mid\left\{\delta ; \exists \alpha \in \Gamma\right.$ ( $\delta$ is the $\gamma$-th element of $\left.\left.A_{\alpha}\right)\right\} \mid=\lambda^{+}$ if there is such a $\gamma$; otherwise put $\gamma=\beta$. If $\gamma<\beta$ let $\delta_{\alpha}$ be the $\gamma$-th element of $A_{\alpha}$ for $\alpha \in \Gamma$, and put $B_{\alpha}=A_{\alpha} \backslash \delta_{\alpha}$; otherwise $\delta_{\alpha}=\lambda^{+}$. It is now easy to find $\Delta \in[\Gamma]^{\lambda^{+}}$such that $B_{\alpha} \cap B_{\beta}=\emptyset$ for distinct $\alpha, \beta \in \Delta$. Set $C=\bigcup_{\alpha \in \Delta} A_{\alpha} \cap \delta_{\alpha}$. In the general case, $\Lambda=\Delta$ and $A=C$ satisfy the requirements of the lemma. In case $\lambda$ is regular and $|C|=\lambda$, we easily find $A \subseteq C$ with $|A|<\lambda$ and $\Lambda \in[\Delta]^{\lambda^{+}}$with $A_{\alpha} \cap \delta_{\alpha} \subseteq A$ for $\alpha \in \Lambda$.

Notice that in case $\lambda^{<\lambda}=\lambda$, the $\Delta$-system lemma says something much stronger [Ku 1, II.1.6]. - We are ready to state and prove the main result of this section.

Theorem 5. Let $\omega_{1} \leq \kappa \leq \lambda \leq 2^{\omega}$ be cardinals, and assume the meager ideal $\mathcal{M}$ on $2^{\omega}$ is $(\kappa, \lambda)$-nicely generated. Then $\kappa=\lambda$. 
Proof. We assume $\kappa<\lambda$ and we seek a contradiction. Without loss $\lambda=\kappa^{+}=2^{\omega}$. Otherwise we force with the collapse Coll $\left(\kappa^{+}, 2^{\omega}\right)=\left\{p ;|p| \leq \kappa\right.$ and $p: \kappa^{+} \rightarrow 2^{\omega}$ is a partial function $\}$. The extension satisfies $|\lambda|=\left|2^{\omega}\right|=\kappa^{+}$, and $\mathcal{M}$ is $\left(\kappa, \kappa^{+}\right)$-nicely generated (because no reals and no Borel sets are adjoined).

Let $\left\langle X_{\alpha} ; \alpha<\kappa^{+}\right\rangle \subseteq P\left(2^{\omega}\right)$ witness that $\mathcal{M}$ is $\left(\kappa, \kappa^{+}\right)$-nicely generated. Let $\left\langle M_{\alpha} ; \alpha<\kappa^{+}\right\rangle$be a sequence of models of $Z F C$ satisfying $\left|M_{\alpha}\right|=\kappa, M_{\alpha} \subseteq M_{\beta}$ for $\alpha \leq \beta$, and $\bigcup_{\alpha<\kappa^{+}} M_{\alpha} \supseteq 2^{\omega}$, such that there are reals $c_{\alpha} \in M_{\alpha+1}\left(\alpha \in \kappa^{+}\right)$Cohen over $M_{\alpha}$. Such a sequence exists as $\operatorname{cov}(\mathcal{M})=\kappa^{+}$(by Fact 3).

For a real $x \in 2^{\omega}$, let $A_{x}=\left\{f \in 2^{\omega} ; \forall n(x(2 n)=f(2 n))\right\}$ and $B_{x}=\{f \in$ $\left.2^{\omega} ; \forall n(x(2 n+1)=f(2 n+1))\right\}$. The $A_{x}$ 's and $B_{x}$ 's are closed nowhere dense sets. Recall that if $M \subseteq N$ are models of $Z F C, c \in N$ is $\mathbb{C}$-generic over $M$ and $d$ is $\mathbb{C}$-generic over $N$, then $(c, d)$ is $\mathbb{C} \times \mathbb{C}$-generic over $M$, and thus $f=f(c, d)$ defined by $f(2 n)=c(2 n)$ and $f(2 n+1)=d(2 n+1)$ is $\mathbb{C}$-generic over $M$ as well (see $[\mathrm{Ku} 2]$ for details).

We put $A_{\alpha}=A_{c_{\alpha}}, B_{\alpha}=B_{c_{\alpha}}$ and $D_{\alpha}=\left\{\beta<\kappa^{+} ; A_{\alpha} \cap X_{\beta} \neq \emptyset\right\}$ for $\alpha<\kappa^{+}$. By assumption $\left|D_{\alpha}\right|<\kappa$, and we can apply Lemma 4 to the family $\left\{D_{\alpha} ; \alpha \in \kappa^{+}\right\}$to get $\Lambda \in\left[\kappa^{+}\right]^{\kappa^{+}}$and $D$ of size $\leq \kappa$ with $D_{\alpha} \cap D_{\beta} \subseteq D$ for distinct $\alpha, \beta \in \Lambda$. For $\alpha \in D$ find a Borel meager set $S_{\alpha}$ containing $X_{\alpha}$. The $S_{\alpha}$ are coded by reals, so there is $\beta_{0} \in \kappa^{+}$such that $M_{\beta_{0}}$ contains all $S_{\alpha}$, where $\alpha \in D$. Let $\left\langle\beta_{\alpha} ; 0<\alpha \leq \kappa\right\rangle$ be a strictly increasing sequence of elements of $\Lambda$ larger than $\beta_{0}$. Let $f_{\alpha}=f\left(c_{\beta_{\alpha}}, c_{\beta_{\kappa}}\right)$ for $0 \leq \alpha<\kappa$. Then $\left\{f_{\alpha} ; \alpha<\kappa\right\} \subseteq B_{\beta_{\kappa}}$. Also $f_{\alpha} \in A_{\beta_{\alpha}}$, and thus $f_{\alpha} \in X_{\gamma_{\alpha}}$ for some $\gamma_{\alpha} \in D_{\beta_{\alpha}}$. As $f_{\alpha}$ is Cohen over $M_{\beta_{0}}, f_{\alpha} \notin S_{\gamma}$ - and in particular $f_{\alpha} \notin X_{\gamma}$ - for $\gamma \in D$. Hence $\gamma_{\alpha} \in D_{\beta_{\alpha}} \backslash D$, and therefore the $\gamma_{\alpha}$ 's are distinct for distinct $\alpha$ 's. This entails that $B_{\beta_{\kappa}}$ is not included in the union of less than $\kappa$ many $X_{\delta}$ 's, a contradiction.

Theorem $5^{\star}$. Let $\omega_{1} \leq \kappa \leq \lambda \leq 2^{\omega}$ be cardinals, and assume the null ideal $\mathcal{N}$ on $2^{\omega}$ is $(\kappa, \lambda)$-nicely generated. Then $\kappa=\lambda$.

Proof. Simply replace all instances of Cohen, $\mathbb{C}, \mathbb{C} \times \mathbb{C}$, and meager by random, $\mathbb{B}$, $\mathbb{B} \star \mathbb{B}$ (where $\star$ denotes iteration), and null in the above proof - and notice that the sets $A_{x}$ and $B_{x}$ defined there are null as well.

Corollary 6. Assume $\kappa$ is regular. Then the meager ideal $\mathcal{M}$ on $2^{\omega}$ is $(\kappa, \lambda)$-nicely generated for some $\lambda \geq \kappa$ iff $\operatorname{add}(\mathcal{M})=\operatorname{cof}(\mathcal{M})=\kappa$. A dual statement holds for the null ideal $\mathcal{N}$.

Proof. $(\Longrightarrow)$ Theorem $5\left(5^{\star}\right)$ and Fact 3 .

$(\Longleftarrow)$ Any ideal $\mathcal{I}$ with $\operatorname{add}(\mathcal{I})=\operatorname{cof}(\mathcal{I})=\kappa$ is easily seen to be $(\kappa, \kappa)$-nicely generated.

We do not know what happens in the singular case. It might be consistent that, for some singular $\kappa, \mathcal{M}$ is $(\kappa, \kappa)$-nicely generated in which case we would have $\operatorname{add}(\mathcal{M})=\operatorname{cov}(\mathcal{M})=c f(\kappa)$, unif $(\mathcal{M})=\kappa$, and $\operatorname{cof}(\mathcal{M})=\operatorname{cov}^{\star}(<\kappa, \kappa)>\kappa($ Fact $3)$. We conjecture, however, that this is not the case.

Our result answers a question addressed by Jacek Cichoń (see [Mi, problem 15.2]). In case $\kappa=\omega_{1}$ Theorem 5 (or Corollary 6 ) shows that the real line cannot be the disjoint union of $\omega_{2}$ meager sets such that every meager set is contained in a countable union of them. 


\section{Chaotic ideals}

Definition 7. Let $X, Y$ be sets and $\mathcal{I} \subseteq P(X), \mathcal{J} \subseteq P(Y)$ be ideals. We say $\mathcal{I}$ and $\mathcal{J}$ are isomorphic $(\mathcal{I} \cong \mathcal{J})$ if there is a bijection $f: X \rightarrow Y$ such that $A \in \mathcal{I}$ iff $f(A) \in \mathcal{J}$ for all $A \subseteq X$.

Let $\mathcal{I}_{0}$ be the ideal on $\omega_{2} \times \omega_{2}$ generated by rectangles of the form $A \times \omega_{2}$, where $A \subseteq \omega_{2}$ is countable. This is a typical example of an $\left(\omega_{1}, \omega_{2}\right)$-nicely generated ideal. Originally, Cichoń conjectured that adding $\omega_{2}$ Cohen reals to a model of $C H$ forces $\mathcal{M}$ to be isomorphic to $\mathcal{I}_{0}$. This is false by Theorem 5 . However one may still ask whether there is a reasonably nice $\sigma$-ideal $\mathcal{I}$ on $\omega_{2}$ (or $\omega_{2} \times \omega_{2}$ or some other underlying set of size $\omega_{2}$ ) such that $\mathcal{M}$ is isomorphic to $\mathcal{I}$ in the Cohen model. Of course, what is meant by reasonably nice should be clarified. One way of doing this requires that such an ideal exist (i.e. be definable) in any $Z F C$-model (as does the above example $\mathcal{I}_{0}$ ). Certainly, it won't be the same in different models; however, when one steps from some model $V$ into a $c c c$ forcing extension $W$, any $\sigma$-ideal of $V$ still generates a $\sigma$-ideal in $W$. This leads to the following notion.

Definition 8. Let $V \subseteq W$ be models of $Z F C$ such that $W$ is a $c c c$ forcing extension of $V$. Let $X \in W$, and let $\mathcal{J} \subseteq P(X)$ with $\mathcal{J} \in W$ be a $\sigma$-ideal. We say $\mathcal{J}$ is chaotic over $V$ if for every $\sigma$-ideal $\mathcal{I} \subseteq P\left(|X|^{W}\right)$ with $\mathcal{I} \in V$ we have $\mathcal{J} ¥ \mathcal{I}^{W}$, where $\mathcal{I}^{W}$ denotes the ideal generated by $\mathcal{I}$ in $W$.

Theorem 9. Let $\kappa \geq \omega_{2}$. Then

$$
\|_{\mathbb{C}_{\kappa}} \text { "M is chaotic over } V " .
$$

Proof. Let $\mathcal{I}$ be a $\sigma$-ideal on $\lambda$ in $V$ where $\| \mathbb{C}_{\kappa} " 2^{\omega}=\lambda "$. We have to show that the ideal generated by $\mathcal{I}$ in the generic extension is not isomorphic to $\mathcal{M}$. Assume the contrary, and let $\dot{f}$ be a $\mathbb{C}_{\kappa}$-name for a bijection between $\lambda$ and $2^{\omega}$ giving rise to an isomorphism.

Let $\left\langle Y_{n} ; n \in \omega\right\rangle$ be a partition of $\omega$ into infinitely many infinite sets. For a real $x \in 2^{\omega}$, let $A_{x}=\left\{f \in 2^{\omega} ; \exists n \in \omega\left(f\left\lceil Y_{n}=x\left\lceil Y_{n}\right)\right\}\right.\right.$. The $A_{x}$ 's are meager (and null). Let $\dot{c}_{\alpha}$ be the name for the $\alpha$-th Cohen real $(\alpha \in \kappa)$, and $\dot{A}_{\alpha}$ denotes the name for $A_{\dot{c}_{\alpha}}$. For each $\alpha$ we can find a name for a meager Borel set $\dot{M}_{\alpha}$ and a set $X_{\alpha} \in \mathcal{I}$ such that

$$
\|_{\mathbb{C}_{\kappa}} " \dot{A}_{\alpha} \subseteq \dot{M}_{\alpha} \wedge \dot{f}\left(X_{\alpha}\right)=\dot{M}_{\alpha} "
$$

To see this fix $\alpha \in \kappa$. As $\|-\mathbb{C}_{\kappa}$ " $\dot{A}_{\alpha}$ is meager", find $X_{\alpha}^{0} \in \mathcal{I}$ with $\|-\mathbb{C}_{\kappa}$ " $\dot{A}_{\alpha} \subseteq$ $\dot{f}\left(X_{\alpha}^{0}\right)$ ", using the $c c c$-ness of $\mathbb{C}_{\kappa}$ and the fact that $\mathcal{I}$ is a $\sigma$-ideal. Let $\dot{M}_{\alpha}^{0}$ be a name for a meager Borel set such that $\|-\mathbb{C}_{\kappa} " \dot{f}\left(X_{\alpha}^{0}\right) \subseteq \dot{M}_{\alpha}^{0} "$. Iterating this construction find $X_{\alpha}^{n} \in \mathcal{I}$ and names for meager Borel sets $\dot{M}_{\alpha}^{n}$ with $\|-\mathbb{C}_{\kappa} " \dot{f}\left(X_{\alpha}^{n}\right) \subseteq \dot{M}_{\alpha}^{n} \subseteq$ $\dot{f}\left(X_{\alpha}^{n+1}\right)$ ". In the end, put $X_{\alpha}=\bigcup_{n \in \omega} X_{\alpha}^{n}$ and let $\dot{M}_{\alpha}$ be a name for the union of the $\dot{M}_{\alpha}^{n}$.

As each $\dot{M}_{\alpha}$ is essentially a real, we can find countable sets $\{\alpha\} \subseteq D_{\alpha} \subseteq \kappa$ such that the interpretation of $\dot{M}_{\alpha}$ lies already in the intermediate extension via the complete subalgebra $\mathbb{C}_{D_{\alpha}}$. Applying Lemma 4 to $\omega_{1}$ and the $D_{\alpha}$, we find $\Lambda \in[\kappa]^{\omega_{2}}$ and $D \subseteq \kappa$ countable $\left(|D| \leq \omega_{1}\right.$ suffices) such that $D_{\alpha} \cap D_{\beta} \subseteq D$ for distinct $\alpha, \beta \in \Lambda$. Without loss $D \neq \emptyset$.

Step into the intermediate extension $V^{\prime}$ via $\mathbb{C}_{D}$; and let $\left\{\alpha_{n} ; n \in \omega\right\} \subseteq \Lambda \backslash D$ (in $V)$. For $\beta \in \lambda$, let $x_{\beta}=\left\{n \in \omega ; \beta \in X_{\alpha_{n}}\right\} ; x_{\beta}$ is (essentially) a real lying 
in the ground model $V$. Let $x \in V^{\prime} \backslash V$ be a subset of $\omega$, and let $\phi \in \omega^{\omega}$ be the increasing enumeration of $x$. Recall that $\mathbb{C}_{\kappa} \cong \mathbb{C}_{D} \times \mathbb{C}_{\kappa \backslash D}[\mathrm{Ku} 2]$, and let $\dot{c}$ be the $\mathbb{C}_{\kappa \backslash D^{-}}$name for the following real (in the model $V^{\prime}$ ):

$$
\Vdash \mathbb{C}_{\kappa \backslash D} " \dot{c}\left\lceil Y_{n}=\dot{c}_{\alpha_{\phi(n)}}\left\lceil Y_{n} " .\right.\right.
$$

Thus $\dot{c}$ is forced to be an element of $\dot{A}_{\alpha_{n}}$ (and hence of $\dot{M}_{\alpha_{n}}$ ) for $n \in x$. On the other hand, the interpretation of $\dot{c}$ is still Cohen over the extension via $\mathbb{C}_{D_{\alpha_{n}} \backslash D}$ for $n \notin x$; in particular $\dot{c}$ is forced to avoid $\dot{M}_{\alpha_{n}}$ for $n \notin x$. Hence

$$
\|_{\mathbb{C}_{\kappa \backslash D}} " \dot{f}^{-1}(\dot{c}) \in \bigcap_{n \in x} X_{\alpha_{n}} \cap \bigcap_{n \notin x}\left(\lambda \backslash X_{\alpha_{n}}\right) " .
$$

This is a contradiction because $\beta \in \bigcap_{n \in x_{\beta}} X_{\alpha_{n}} \cap \bigcap_{n \notin x_{\beta}}\left(\lambda \backslash X_{\alpha_{n}}\right)$ and $x_{\beta} \neq x$ for all $\beta \in \lambda$.

Notice that Theorem 9 provides an alternative argument for Cichoń's original conjecture to be false in the Cohen real model. We also note that Theorem 9 has the following funny consequence: assume we add first $\omega_{2}$ Cohen reals and then again $\omega_{2}$ Cohen reals; let $\mathcal{M}^{\prime}$ be the meager ideal in the intermediate model, and $\mathcal{M}$ the meager ideal in the final model; let $\mathcal{M}^{\star}$ be the ideal generated by $\mathcal{M}^{\prime}$ in the second extension. Then $\mathcal{M}^{\star} \neq \mathcal{M}$, although they have the same cardinal characteristics and are intuitively similar because they are gotten by the same kind of extension. - As in the case of Theorem 5 , it is immediate that the dual statement about the null ideal can be proved in exactly the same way.

Theorem $\mathbf{9}^{\star}$. Let $\kappa \geq \omega_{2}$. Then

$$
\|_{\mathbb{B}_{\kappa}} \text { "N } \mathcal{N} \text { is chaotic over } V \text { ". }
$$

We now switch to the investigation of the meager ideal in the random extension (and, dually, the investigation of the null ideal in the Cohen extension).

Theorem 10. Let $\kappa \geq \omega_{2}$. Then

$$
\|_{\mathbb{B}_{\kappa}} \text { "M is chaotic over } V \text { ". }
$$

Proof. We start as in the proof of Theorem 9 with a $\sigma$-ideal $\mathcal{I}$ on $\lambda$ in $V$, where $\|_{\mathbb{B}_{\kappa}} " 2^{\omega}=\lambda "$, and a $\mathbb{B}_{\kappa}-$ name $\dot{f}$ for a bijection between $\lambda$ and $2^{\omega}$.

Next recall that $\mathbb{B}$ adjoins a meager Borel set containing all ground model reals [Ku 2]. Let $\dot{A}_{\alpha}$ be a $\mathbb{B}_{\{\alpha\}}-$ name for such a set. We find as before $\mathbb{B}_{\kappa}-$ names $\dot{M}_{\alpha}$ for meager Borel sets and $X_{\alpha} \in \mathcal{I}$ such that

$$
\|_{\mathbb{B}_{\kappa}} \text { " } \dot{A}_{\alpha} \subseteq \dot{M}_{\alpha} \wedge \dot{f}\left(X_{\alpha}\right)=\dot{M}_{\alpha} " .
$$

The next paragraph in the proof of Theorem 9 can be taken over with $\mathbb{C}$ replaced by $\mathbb{B}$ : we get the $D_{\alpha}$ 's, $\Lambda \in[\kappa]^{\omega_{2}}$ and $D$.

Then we step again into the intermediate extension $V^{\prime}$ via $\mathbb{B}_{D}$, and choose $\left\{\alpha_{n} ; n \in \omega\right\} \subseteq \Lambda \backslash D$. The $x_{\beta}, \beta<\lambda$, and $x$ are as before. Next we step into the extension $V^{\prime \prime}$ of $V^{\prime}$ via $\mathbb{B}_{\cup_{n \notin x} D_{\alpha_{n}} \backslash D}$. In this model find a real $d$ which is not in the interpretation of any $\dot{M}_{\alpha_{n}}, n \notin x$. On the other hand, $d$ is forced to lie in $\dot{A}_{\alpha_{n}}$ for $n \in x$ (in fact, this is true for any real of $V^{\prime \prime}$ ); in particular it will be in $\dot{M}_{\alpha_{n}}$ for $n \in x$. Thus we have again

$$
\|_{\mathbb{B}_{\kappa} \backslash D} " \dot{f}^{-1}(\dot{d}) \in \bigcap_{n \in x} X_{\alpha_{n}} \cap \bigcap_{n \notin x}\left(\lambda \backslash X_{\alpha_{n}}\right) ",
$$


where $\dot{d}$ is the $\mathbb{B}_{\kappa \backslash D}$-name for $d$. This is a contradiction as in the proof of Theorem 9.

An exactly similar argument yields:

Theorem $10^{\star}$. Let $\kappa \geq \omega_{2}$. Then

$$
\Vdash_{\mathbb{C}_{\kappa}} \text { "N is chaotic over } V " \text {. }
$$

We have seen several situations in which $\mathcal{M}$ and $\mathcal{N}$ are chaotic, but the problem remains whether they can be non-chaotic in a non-trivial way. Of course, if the $c c c$-extension forces $M A$ to hold, then $\mathcal{M}$ and $\mathcal{N}$ are not chaotic over the ground model. However, in this case, $\operatorname{cof}(\mathcal{M})=\operatorname{cof}(\mathcal{N})=\operatorname{add}(\mathcal{M})=\operatorname{add}(\mathcal{N})=2^{\omega}$, and the structure of both ideals is well known and trivial. One may still hope that in other models of $Z F C$ (e.g. the ones gotten from adding iteratively $\omega_{2}$ Laver or $\omega_{2}$ Miller reals with countable support over a model for $C H$ ), $\mathcal{M}$ and $\mathcal{N}$ have a nicer structure. Note in this context that $c c c$ can be replaced by proper and cardinal-preserving in Definition 8.

Note added to the revised version. After having seen our present work, Janusz Pawlikowski has found a purely combinatorial result concerning products of ideals which easily entails our Theorem 5 .

\section{REFERENCES}

[BJ] T. Bartoszyński and H. Judah, Set Theory, On the Structure of the Real Line, A. K. Peters, Wellesley, MA, 1995.

[BJS] T. Bartoszyński, H. Judah and S. Shelah, The Cichon diagram, Journal of Symbolic Logic 58 (1993), 401-423. MR 94m:03077

[Fr] D. Fremlin, Cichon's diagram, Séminaire Initiation à l'Analyse (G. Choquet, M. Rogalski, J. Saint Raymond, eds.), Publications Mathématiques de l'Université Pierre et Marie Curie, Paris, 1984, pp. 5-01-5-13.

[Je] T. Jech, Set theory, Academic Press, New York, 1978. MR 80a:03062

[Ku 1] K. Kunen, Set theory, North-Holland, Amsterdam, 1980. MR 82f:03001

$[\mathrm{Ku} 2]$ K. Kunen, Random and Cohen reals, Handbook of set-theoretic topology (K. Kunen and J. Vaughan, eds.), North-Holland, Amsterdam, 1984, pp. 887-911. MR 86d:03049

[Mi] A. Miller, Arnie Miller's problem list, in: Set Theory of the Reals (H. Judah, ed.), vol. 6, Israel Mathematical Conference Proceedings, 1993, pp. 645-654. MR 94m:03073

Mathematisches Institut der Universität Tübingen, Auf Der Morgenstelle 10, 72076 Tübingen, Germany

E-mail address: jobr@michelangelo.mathematik.uni-tuebingen.de

Current address: Department of Mathematics, Bradley Hall, Dartmouth College, Hanover, New Hampshire 03755

E-mail address: brendle@mac.dartmouth.edu 